\title{
Findings About Events or Interventions Location
}

National Cancer Institute

\section{Source}

National Cancer Institute. Findings About Events or Interventions Location. NCI

Thesaurus. Code C113363.

The physical portion of the body at which the findings about the event or intervention assessment takes place. 